\title{
Using hemodynamic parameters quantified with computational fluid dynamics to explore potential mechanisms of occurrence and progression in Alzheimer Disease
}

\section{Jian Xie}

Department of Neurology, Affiliated Zhongda Hospital『School of Medicine区Southeast University

\section{Zaiheng Cheng}

Shenzhen Institutes of Advanced Technology $₫$ Chinese Academy of Science

\section{LiHua Gu}

Department of Neurology $₫$ Affiliated ZhongDa Hospital『School of Medicine $\bigotimes$ Southeast University

\section{Bokai Wu}

Shenzhen Institutes of Advanced Technology Chinese Academy of Sciences

\section{Gao-jia Zhang}

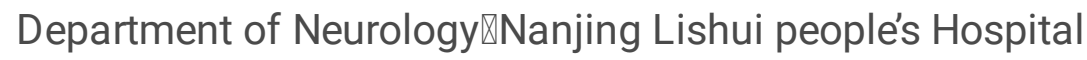

\section{Wen-Shin Shiu}

Shenzhen Institutes of Advanced Technology Chinese Academy of Sciences

\section{Rongliang Chen}

Shenzhen Institutes of Advanced Technology Chinese Academy of Sciences

\section{Zan Wang}

Department of Neurology, Affiliated ZhongDa Hospital『School of Medicine『Southeast University

\section{Chang Liu}

Shenzhen Institutes of Advanced Technology Chinese Academy of Sciences

\section{Xiao-chuan Cai}

Shenzhen Institutes of Advanced Technology Chinese Academy of Sciences

\section{Li-Ping Wang}

Shenzhen Institutes of Advanced Technology Chinese Academy of Sciences

Jia Liu ( $\square$ jia.liu@siat.ac.cn )

Shenzhen Institutes of Advanced Technology Chinese Academy of Sciences

Zhijun Zhang ( $\nabla$ janemengzhang@vip.163.com )

Southeast University https://orcid.org/0000-0001-5480-0888

\section{Research}


Keywords: Computational fluid dynamics, Hemodynamics, Cerebral blood flow, Cerebrovascular resistance, Alzheimer's disease

Posted Date: June 7th, 2020

DOI: https://doi.org/10.21203/rs.3.rs-32881/v1

License: (c) (1) This work is licensed under a Creative Commons Attribution 4.0 International License. Read Full License 
Using hemodynamic parameters quantified with computational fluid dynamics to explore potential mechanisms of occurrence and progression in Alzheimer Disease

Jian Xie ${ }^{\S 1}$, Zaiheng Cheng ${ }^{\S 2}$, LiHua Gu${ }^{1}$, Bokai Wu² , Gao-jia Zhang ${ }^{3}$,Wen-Shin Shiu ${ }^{2}$, Rongliang Chen ${ }^{2}$, Zan Wang ${ }^{1}$, Chang $\mathrm{Liu}^{2}$, Xiao-chuan Cai ${ }^{4}$, Li-Ping Wang ${ }^{2}$, Jia Liu*2, Zhijun Zhang ${ }^{1 *}$

$\S$ These authors contributed equally to this work.

*Correspondence: janemengzhang@vip.163.com; jia.liu@siat.ac.cn.

${ }^{1}$ Zhijun Zhang, PhD\& MD, Department of Neurology, Affiliated ZhongDa Hospital, School of Medicine, Southeast University, Nanjing, Jiangsu 210009, China.

${ }^{2}$ Jia Liu, PhD, Shenzhen Institutes of Advanced Technology, Chinese Academy of Sciences, Shenzhen, Guangzhou 518055, China.

Full list of author information is available at the end of the article

\section{Abstract}

Background: It has gradually recognized that the patients with Alzheimer's disease (AD) have cerebral hemodynamic disorders. The purpose of the present study was to exploit a novel computational fluid dynamics (CFD) model, which could be used to measure intracranial hemodynamics quantitively in $\mathrm{AD}$ patients and to further explore how the hemodynamic changes are involved in progression of AD.

Methods: A novel CFD model was constructed by personal magnetic resonance angiography (MRA), vessel ultrasound and blood pressure value of all subjects, of whom included $\mathrm{AD}$ patients, vascular dementia (VaD) patients and well-matched 
healthy controls (HCs). Demographic, clinical and imaging data of all subjects were recorded and analyzed. Quantitative total cerebral blood flow (CBF) and cerebrovascular resistance (CVR) were compared among three groups, in order to ascertain the potential hemodynamic disorders in $\mathrm{AD}$ patients.

Results: Total CBF and CVR of AD patients were significantly different from those of HCs (both $P<0.01$ ), but not different from patients with $\mathrm{VaD}$ (both $P>0.5$ ), despite the cerebral arteries in $\mathrm{AD}$ patients were anatomically intact. Total $\mathrm{CBF}$ was negatively correlated with total CVR $\left(r_{s}=-0.822, P<0.001\right)$ in AD patients. Comparing with HCs, Elevated CVR $(O R=2.25, P=0.004)$ and age ( $O R=2.06, P=0.021)$ were independent risk factor of $\mathrm{AD}$.

Conclusions: CFD can be applied to non-invasively and conveniently quantify and visualize biomechanical changes of cerebral blood flow. Patients with AD have dysfunction of cerebral hemodynamic, including lower CBF and higher CVR, and the CVR was an independent risk factor of $\mathrm{AD}$. These findings provide quantitative evidence to support that increase of cerebrovascular resistance may involve in development of AD.

Key words: Computational fluid dynamics, Hemodynamics, Cerebral blood flow, Cerebrovascular resistance, Alzheimer's disease.

\section{Introduction}

Dementia is a disorder characterized by the impairment of cognitive function with attenuated daily activity and psychiatric symptoms. Dementia is the third contributor of neurological disability-adjusted life-years (DALYs) ${ }^{[1]}$. More than 50 million people are 
affected by the dementia globally, it has been estimated that the total number of dementia patients worldwide will reach 76 million by 2030 and 135 million by $2050^{[2]}$. $\mathrm{AD}$ and $\mathrm{VaD}$ are the most common causes of dementia ${ }^{[3]}$. As acknowledged by clinicians, lifestyle and vascular risk factors accelerate VaD progression ${ }^{[4]}$. However, recently studies indicated that cardiovascular risk factors correlate with the occurrence and development of $\mathrm{AD}^{[5]}$. For example, previous studies have confirmed higher vascular risk and lower physical activity are associated with burden of $\beta$-Amyloid and cognitive decline ${ }^{[6,7]}$.

The circulatory pathophysiological changes mediated by vascular risk factors were always accompanied by intracranial hemodynamic disorder, which was involved in mechanisms of cognitive decline ${ }^{[5-7]}$. For instance, patients with cardiac dysfunction manifest hemodynamic disorders and decreased cerebral perfusion, which subsequently lead to injury or death of neurons ${ }^{[8,9]}$. Moreover, remodeling and cerebral vasomotor disorders of intracranial or extracranial vessels reduce cerebral perfusion and increase resistance of cerebral arteries, which impair metabolism of nervous tissue and clearance of A- $\beta$ amyloid, further exacerbate cognitive decline ${ }^{[10-12]}$. Therefore detection of hemodynamic disorders may contribute to identification potentially pathophysiological changes in dementia patients.

Hemodynamic parameters can be measured indirectly through some medical imaging techniques, including arterial spin labeling (ASL) MRI, transcranial doppler ultrasonography (TCD), oxygen-15-labelled water positron emission tomography (PET), four dimensional (4D) flow MRI. Using ASL, the CBF ratio of gray 
matter/white matter has been shown to decline globally in the poststroke dementia patients $^{[13]}$, AD patients also exhibit increased CVR index (CVRi) and diminished CBF in inferior parietal and temporal cerebral ${ }^{[14,15]}$. However, ASL has poor noise to signal ratio and only reflects changes in a small portion of hemodynamic parameters. Flow velocity and the pulsatility index can be evaluated by TCD, in which, increased CVRi were found in aged adults ${ }^{[16]}$ and $\mathrm{AD}$ patients ${ }^{[17]}$. A meta-analysis indicated that hemodynamic disturbance in $\mathrm{VaD}$ was more severe than that of $\mathrm{AD}^{[18]}$. However, $\mathrm{TCD}$ cannot accurately detect hemodynamic parameters of distal arterial branches, furthermore, the accuracy of TCD relies on an experienced operator and interpreter. PET only measures the CBF and is applied limitedly. The 4D flow MRI is an emerging imaging paradigm and capable to quantify the temporal evolution of complex blood flow patterns within an acquired $3 \mathrm{D}$ volume, by which $\mathrm{AD}$ patients have been found to have decreased mean flow in the internal carotid and middle cerebral arteries ${ }^{[19]}$. However, there is a trade-off between the spatial and temporal resolution of $4 \mathrm{D}$-flow MRI, it is suitable either for the large arteries with fast velocity or the narrow vessels with slow velocity, such as measurements of blood flow velocity in the aorta or veins, thereby limiting the applications of 4D-flow MRI in cerebral arteries.

CFD is a well-established technique that provides comprehensive information of hemodynamics non-invasively. Various 3D CFD models using routinely available medical imaging had been proposed and applied to evaluate hemodynamic parameters, for example, fractional flow reserve (FFR) was calculated by CFD based on computed tomography angiography (CTA), which has been approved to assess the risk of 
coronary stenosis, and CFD derived FFR is highly comparable with the FFR measured by a interventional pressure wire ${ }^{[20,21]}$. CFD technique can reduce unnecessary interventional angiography effectively and help doctors to diagnose pathological conditions $^{[22,23]}$. Moreover, CFD can be applied to assess the risk of rupture and pressure of the intracranial aneurysm, thereby improving the understanding of the biomechanics of the aneurysms ${ }^{[24]}$.

To our knowledge, there is lack of study on hemodynamic alterations in AD patients using CFD. The present study would use self-constructed CFD model to quantify the hemodynamic parameters and compared among three groups: (1) AD patients, (2)VaD patients as positive controls and (3)HCs as negative controls, so as to explore potential mechanisms of occurrence and progression in Alzheimer Disease.

\section{Methods}

\section{Participants}

The present cross-sectional study included $\mathrm{AD}$ patients $(\mathrm{n}=30), \mathrm{VaD}$ patients $(n=29)$, and HCs $(n=34)$. Probable AD diagnosis was determined in accordance with the criteria of the National Institute of Neurological and Communicative Disorders and Stroke, and the AD and Related Disorders Association (NINCDS-ADRDA). Probable VaD was diagnosed in accordance with the criteria of the International Classification of Diseases-10 (ICD-10). Individuals who were cognitively normal were also included to be HCs. All participants received MRI+MRA and ultrasound of cervical arteries.

Subjects were excluded from the study if they suffered from heavy organ dysfunction, or a history of cognitive disorders. The study was approved by the ethics committee of 
the affiliated ZhongDa hospital of Southeast University.

\section{Collection of demographic data}

All participants underwent comprehensive medical and neurological evaluations, fasting venous blood samples were collected for routine blood testing and blood biochemical parameters (Table 1 and Supplementary Table S1). The 10-year risk of heart disease or stroke was determined using the ASCVD algorithm (website: http://www.cvriskcalculator. com/), which was used to evaluated the risk factor burden of cardiovascular and cerebrovascular diseases, ASCVD scores were categorized as low, moderate and high risk depending on the risk stratification. Mean arterial pressure (MAP) was calculated by formula: $\mathrm{MAP}=\mathrm{DBP}+(\mathrm{SBP}-\mathrm{DBP}) / 3$.

\section{Protocols of imaging}

The systolic and diastolic BP of participant were measured prior to examination of cervical vessel ultrasound in the same morning. Doppler ultrasonography was performed to measure velocities of the left and right internal carotid and vertebral arteries (CCA/VA) using high-resolution ultrasound (GE, LOGIQ E9) at 8-15 MHz, in which, peak systolic velocity (PSV) and end diastolic velocity (EDV) acted as two important indexes to build CFD model. All patients were scanned using a 3T clinical MRI system (Siemens) with a 12-channel head and neck coil array. The MR scan included parenchymal brain imaging sequences (axial DWI, T2 FLAIR, and T1), MRA was performed on axial 3D TOF MRA $(\mathrm{TR}=15.0 \mathrm{~ms}, \mathrm{TE}=3.45 \mathrm{~ms}$, flip angle $=25$, $\mathrm{NEX}=1$, field of view $=242 \times 242 \mathrm{~mm}$, matrix size $512 \times 512,24$ slices $\times 3$ sections, slice thickness $1 \mathrm{~mm})$. 
Hemodynamic measurement of subjects using CFD

134

\section{Image processing and CFD mesh generation}

MRA images were exported from computing the server of the MRI scanner in standard Digital Imaging and Communication in Medicine (DICOM) format. The cerebral artery was segmented from each DICOM image using 3D region-growing provided by Mimics (Materialise NV, Belgium), in which results were inspected and refined by two radiologists. The 3D surface of the cerebral artery was then reconstructed. The computational domain of CFD was defined by a mesh generated by ANSYS ICEM CFD software (ANSYS, Inc., USA). Due to the complexity of the geometry, an unstructured tetrahedral cell was used for domain discretization. The total number of elements was greater than 1 million with a minimum volume of approximately $1.0^{*} 10^{-}$ ${ }^{8} \mathrm{~cm}^{3}$ in order to capture features of flow dynamics in small-scale, to provide more detailed computation of the hemodynamics, especially within the stenotic artery.

\section{Modelling of blood flow in 3D}

The blood flow was assumed to be a viscous and incompressible Newtonian fluid, the heat transfer and compressibility effects of the vascular wall were neglected in this process. The blood flow were defined as a constant density $\rho=1.06 \times 10^{3} \mathrm{~kg} \cdot \mathrm{m}^{-1}$ and dynamic viscosity $\mu=3.5 \times 10^{-3} \mathrm{~kg} \cdot \mathrm{m}^{-1} \cdot \mathrm{s}^{-1}$, as the simulated blood flow was not sensitive to these parameters ${ }^{[25,26]}$. A typical carotid artery diameter $D=$ $6.0 \times 10^{-3} \mathrm{~m}$ and its corresponding velocity of blood flow $v=0.4 \mathrm{~m} . \mathrm{s}^{-1}$ were assumed in order to calculate the Reynolds number: $R e=\rho v D / \mu \approx 121$, which suggested that the blood flow was laminar. A 3D unsteady incompressible Navier- 
Stokes equation was then utilized to describe the blood flow, as follows:

$$
\mathrm{U} \frac{\partial \mathbf{v}}{\partial t}+(\mathbf{v} \cdot \boldsymbol{\nabla}) \mathbf{v}=-\frac{1}{\rho} \boldsymbol{\nabla} p+\frac{\mu}{\rho} \nabla^{2} \mathbf{v}+\mathbf{f}
$$

The equation for conservation of mass was defined as:

$$
\boldsymbol{\nabla} \cdot \mathbf{v}=\mathbf{0}
$$

where $\mathbf{v}$ was the velocity vector, $p$ was the pressure, and $\mathbf{f}$ was force of the body, assumed equal to 0 .

To solve equations (1.1) and (1.2), a finite volume approach using ANSYS CFX software version 14.5 (ANSYS, Inc., USA) was used. CFD simulations were conducted on an AMAX server with dual 22-core Intel Xeon E5-2699 v4 CPUs running at 2.20GHz with 256GB memory. Mesh partitioning was performed using a k-way Metis algorithm with a message passing interface (MPI) unutilized for multi-core communication. A five second period of blood flow in each cerebral artery was simulated with a time step of $0.01 \mathrm{~s}$. A second-order backward Euler scheme was used for the transient term. The criteria for convergence was set at a root mean square error (RMSE) for the relative levels of $1.0 \times 10^{-5}$.

\section{Determination of Boundary conditions}

Both PSV and EDV at each internal carotid artery (ICA) and vertebral artery (VA) were used as the inlet boundary conditions to estimate the respective mean velocities, as $V_{\text {mean }}=\frac{1}{3} V_{P S V}+\frac{2}{3} V_{E D V}$. The mean velocities were assumed to be present at the centerline of the vessels, the flow was further assumed to be laminar with pulsatility neglected at all inlets. Inlet blood flow was then approximated by $Q_{\text {in }}=\frac{1}{2} V_{\text {mean }} \cdot A_{\text {in }}$, 
where $A_{\text {in }}$ represents the cross-sectional area of the artery at the inlet, as the

177 hemodynamic assumption resulted in a Poiseuille velocity profile, which is parabolic ${ }^{[27]}$.

178 The cross-sectional area was calculated by $A_{i n}=\pi \cdot\left(\frac{D_{i n}}{2}\right)^{2}$, where $D_{i n}$ was the 179 diameter of the inlet artery, measured from the MRA images. Total CBF was 180 preliminarily obtained from the sum of internal carotid and vertebral $Q_{\text {in. }}$ For the outlet 181 boundary conditions, pressure $P_{\text {out }}$ was estimated at each outlet. A resistive boundary 182 condition was applied to each outlet of the distal artery to mimic the downstream 183 resistance, assumed to be inversely proportional to the diameter of the outlet. In order 184 to achieve this, total CVR $R_{\text {total }}$ was calculated from the total inflow $Q_{\text {total }}=$ $185 Q_{i n}^{I C A}+Q_{i n}^{V A}$ and mean arterial pressure (MAP), approximated by brachial blood 186 pressure. Initial $R_{\text {total }}$ was then calculated from $R_{\text {total }}=M A P / Q_{\text {total }} . R_{\text {out }}$ at each 187 outlet was estimated from $R_{\text {total }}$ depending on the diameter of the outlet $\left(D_{\text {out }}\right)$ as 188 calculated from MRA images. Finally, the outlet pressure $P_{\text {out }}$ was calculated by $189 P_{\text {out }}=Q_{\text {out }} \cdot R_{\text {out }}$, where $Q_{\text {out }}$ was the flow rate at each outlet, estimated from the 190 integral of the outlet velocity $V_{\text {out }}$ at the outlet area. Statistical analysis Statistical analyses were performed using SPSS version 25.0 (IBM Corp.). 193 Normality of continuous data was confirmed using a Shapiro-Wilk test, and 194 homogeneity of variance assessed using Levene test. Data are presented as means \pm standard deviation (SD). Categorical data are expressed numerically. Analysis of differences in demographic, clinical characteristics, and CFD among the three groups were conducted using a one-way analysis of variance (ANOVA), and Kruskal-Wallis 
test or $\chi^{2}$ test. Where a significant difference was found, Dunnett's, Pairwise

Comparisons and Bonferroni methods were used to adjust for each two groups

respectively. Differences in CBF or CVR between gender, with or without a history of stroke were analyzed by independent sample $\mathrm{t}$ tests and Kruskale-Wallis test respectively. The correlation between $\mathrm{CBF}, \mathrm{CVR}$ and age were explored using Spearman correlation analyses. To elucidate the independent contributions of hemodynamic parameters to dementia, binary logistic regression analyses were performed for patients and HCs groups, statistically significant independent variables in univariate analysis were included in a binary regression. In these analyses, AD or VaD was the dependent variable, gender, age, history of stroke, CBF and CVR were independent variables. According to the interquartile range of all subjects, CBF and

CVR were divided into four continuous levels (supplementary Table 2), with entry and removal criteria of 0.05 and 0.1 , respectively. For significant findings, odds ratios $(O R)$ were calculated to interpreted the effect on "dementia". $P$-values of $<0.05$ were considered statistically significant.

\section{Results}

Comparison of baseline demographic and clinical characteristics among three groups for the three groups. There were significant differences in age $(F=14.713, P<0.001)$, gender distribution $(\chi 2=13.449, P=0.001)$ and percentage of stroke history $\left(\chi^{2}=12.041\right.$; $P=0.002$ ) among three groups. As compared to $\mathrm{HCs}$, average age of $\mathrm{VaD}$ patients 
$(P<0.001)$ and $\mathrm{AD}$ patients $(P=0.005)$ were older than HCs, however, no significant

221

difference for age was founded between $\mathrm{AD}$ and $\mathrm{VaD}$ patients $(P=0.058)$. As compared with $\mathrm{AD}$ and HCs groups, the proportion of male and history of stroke in $\mathrm{VaD}$ group were significantly increased (both $P<0.05$ ). Additional information for all subjects is displayed in Supplementary Table S1.

\section{Comparison of Hemodynamic parameters among groups}

Three typical color maps of pressure and velocity throughout the arterial tree are displayed in Figure 1 for three subjects: $\operatorname{AD}(a, b), \operatorname{VaD}(c, d)$, and HCs (e, f). both AD and $\mathrm{VaD}$ patients had diminished blood supply even if the arterial trees of $\mathrm{AD}$ patient were anatomically intact. CBF and CVR in arteries that were larger than $0.2 \mathrm{~cm}$ in diameter could be estimated by the CFD model (Supplementary figure S1). The hemodynamic parameters of all subjects were calculated by the 3D CFD model (Table 2). As compared with $\mathrm{HCs}$, there were significant reduced total CBF or increased total CVR in AD group(CBF: $P=0.008$; CVR: $P=0.009)$ and VaD group (CBF: $P=0.002$; CVR: $P=0.001$ ), however no significant difference in the CBF and CVR were founded between $\mathrm{AD}$ and $\mathrm{VaD}$ patients $(\mathrm{CBF}: P=0.905$; $\mathrm{CVR}: P=0.524)$. other hemodynamic parameters of all subjects are displayed in Supplementary Table S2

Figure1 Three typical examples of pressure distribution and stream lines of blood flow velocity are displayed in the first and the second row, respectively. The first column( fig a and $\mathrm{b}$ )is for an $\mathrm{AD}$ patients, the second column (fig $\mathrm{c}$ and $\mathrm{d}$ ) is for a VaD patients, and the third column (fig e and $\mathrm{f}$ ) is for a healthy subject. It is evident that the AD patient and the healthy subject are with intact arterial trees, whereas $\mathrm{VaD}$ the patient is with scarce arterial branches. However, according to computation, 

$\mathrm{ml} / \mathrm{min}$ (healthy subject) respectively.

\section{Interactive associations of the hemodynamic parameters and risk factors}

Bivariate Spearman correlation showed that total CBF was negatively correlated with total CVR in whole subjects (fig $2 \mathrm{a}, r_{s}=-0.826, P<0.001$ ) and AD groups(fig $2 \mathrm{~b}$, $\left.r_{s}=-0.822, P<0.001\right)$. There were significant correlations between age and total CBF

(fig $2 \mathrm{c}, r_{s}=-0.282, P<0.05$ ) or total CVR(fig $2 \mathrm{~d}, r_{s}=0.278, P<0.05$ ), however there was no significant difference in total CVR (fig 2e, $Z=-0.968 ; P=0.333$ ) or $\mathrm{CBF}$ (fig 2f, $t=0.759 ; P=0.450$ ) between male and female subjects. Meanwhile, as compared with subjects without past history of stroke, the subjects with history of stroke have a higher total CVR (fig 2g, $Z=-2.179 ; P=0.029$ ), but not CBF (fig $2 \mathrm{~h}, t=1.793 ; P=0.076$ ).

Figure 2 Interactive associations of the hemodynamic parameters and risk factors, correlation between total CBF and CVR in all subjects (a) and AD group(b), (c) and (d) showed significant correlations between CBF or CVR and age, fig $(e, f)$ showed there were no significant difference of total CVR or CBF between male and female patients, fig(g) indicated there was significant difference of total CVR in patients with stroke or not, but not total CBF fig(h).ns: no significance, $* P<0.05$.

\section{Association between hemodynamic parameters and dementia}

\section{Discussion}


This present study exploited a 3-D CFD model to quantitatively measure the

changes of $\mathrm{CBF}$ and $\mathrm{CVR}$ in $\mathrm{AD}$ patients for the first time. The main findings are summarized as follows. Firstly, as compared with $\mathrm{HCs}$, both total CBF and CVR in AD or VaD groups were significantly changed, no differences were observed in total CBF and CVR between $\mathrm{AD}$ and VaD groups. Secondly, total CBF was negatively correlated with CVR in all subjects. Finally, elevated CVR and age associated with increased risk of $\mathrm{AD}$, suggesting that changed cerebral hemodynamic are present in $\mathrm{AD}$ patients.

It is challenging to measure hemodynamics directly. Previous studies have used other methods to non-invasively quantify the CBF and CVR ${ }^{[15,28-31]}$. In current study, a CFD model was constructed individually by the subject-specific medical images, It is non-invasive and not limited by contraindications of imaging examinations, CTA and DSA data can also be used to replace MRA, hence it is accessible to most medical centers. The model has high spatial resolution, and arteries with diameter larger than $0.2 \mathrm{~cm}$ can be evaluated, allowing hemodynamic parameters even in the distal branches to be available. Furthermore, comprehensive hemodynamic parameters, such as CBF, velocity, CVR, FFR, and arterial wall shear stress can be acquired anywhere of the artery conveniently in the 3D model.

During undertaking cognitive task, healthy subjects and stroke patients exhibited a significant increase both in CBF and blood stream velocity ${ }^{[10,32]}$, which suggested that cerebrovascular circulation adjusts its hemodynamic response to metabolic requirements. However, the total $\mathrm{CBF}$ of the internal carotid and vertebral arteries were decreased in VaD patients ${ }^{[28]}$, Furthermore, a marked decreased CBF in the parietal and 
frontal cortex of $\mathrm{AD}$ or $\mathrm{VaD}$ patients has been observed, which was associated with increased subcortical white matter lesions in VaD patients ${ }^{[33]}$. Stabilized CBF is dependent on heart function and resistance of intracranial vessels[9], the CVRi of middle cerebral arteries ${ }^{[17]}$, cortex and subcortex were increased in $\mathrm{AD}$ patients, particularly within the thalamus and caudate[16, 34]. In addition CVRi was positively correlated with severity of dementia ${ }^{[17]}$. Hence hemodynamic alterations were involved in the pathophysiology of $\mathrm{AD}$, therefore the alterations of vascular resistance may play an important role progression of $\mathrm{AD}$ and $\mathrm{VaD}$. CBF respectively. In present study, the decreased total CBF and increased CVR were importantly, the total CBF was significantly and negatively correlated with total CVR. Therefore, the CBF may be regulated by CVR. All above results demonstrated that the increased by mixed brain lesions and remodeling of cerebral microvasculature which 
include the alleviation of cerebrovascular lesions, careful control or decreased exposure to risk factors may attenuate cognitive decline, and alleviation of the capillary contraction caused by $\mathrm{A} \beta$ may be a new treatment direction of $\mathrm{AD}$.

\section{Limitations}

There are some limitations to this study. Firstly, it is a cross-sectional research study, the correlation between hemodynamic parameters and AD need to be verified by follow-up studies. In a future study we will verify the correlation between more hemodynamic parameters and dementia with follow-up investigation, in addition the effect of hemodynamics on progression. Secondly, the diagnosis of AD was based on clinical data and lack of neuropathic markers. Thirdly, due to the small number of patients, which may restrict findings of this study, and the large-scale clinical studies comparation with other non-invasive methods to explore the practicality of CFD.

\section{Conclusions}

CFD can be used to distinguish hemodynamic changes between AD patients and healthy subjects. AD patients had lower CBF and higher CVR, and the CVR was an independent risk factor of AD, Early detection of alterations of CVR will help clinicians find potential cerebrovascular lesions, alleviation of CVR may be another direction of treatment in $\mathrm{AD}$.

\section{Supplementary information}

Table S1. Supplementary demographics and clinical characteristics of all subjects.

Table S2. Hemodynamic parameters of all subjects. FigureS1. Procedure of CFD model. 


\section{Abbreviations}

331

AD: Alzheimer's disease, ASL: arterial spin labeling, BMI: body mass index, CFD: computational fluid dynamics, CVR: cerebral vascular resistance, CVRi: CVR index, CBF: cerebral blood flow, CHD: coronary heart disease, DBP: diastolic blood pressure, EDV: end diastolic velocity, FFR: fractional flow reserve, GT: triglycerides, HCs: healthy control subjects, HDL: high-density lipoprotein, Hb: hemoglobin, LDL: lowdensity lipoprotein, MAP: mean arterial pressure, MRA: magnetic resonance angiography, PSV: peak systolic velocity, SBP: systolic BP, Tc: total cholesterol, TCD: transcranial doppler ultrasonography, VaD: vascular dementia.

\section{Acknowledgements}

We wish to thank all participants in this study, without whose agreement this research would not have been possible. We thank the colleagues who participated in the collection of the clinical data.

\section{Author's contributions}

ZJZ and JL designed the study, analyzed and interpreted of data, and drafted and revised the manuscript, JL Jia contributed to technique writing. JX collected, analyzed and interpreted the data, prepared all statistic figures, drafted the manuscript. ZC and BW contributed to arterial 3D reconstruction and mesh generation. GJZ, GLH and ZW contributed to collected the clinical data, WSS, RLC and XCC contributed to numerical computation, CL contributed to medical image processing, LPW participated in design the study. All authors contributed to the writing and revisions of the paper and approved the final version. 


\section{Funding}

353 This research was supported by grants from the National Natural Science Foundation 354 of China (81420108012, 81671046 to Z.Z.), Program of Excellent Talents in Medical

355 Science of Jiangsu Province (JCRCA2016006 to Z.Z.). National Natural Science 356 Foundation of China $(81661168015,61703017,81871447$ to JL), and Shenzhen 357 Science and Technology Innovation Commission (ZDSYS201703031711426 to JL).

358 The funding bodies had no role in the design of the study, data collection, ana-lysis, 359 interpretation, or writing of the manuscript.

\section{Availability of data and materials}

The dataset used during the current study is available from the corresponding author on reasonable request.

\section{Ethics approval and consent to participate}

364 The study was approved by the Institutional Ethical Committee of Nanjing ZhongDa 365 Hospital, Southeast University and the participants gave written informed consents 366 prior to obtain the data.

\section{Consent for publication}

368 Not applicable

\section{Competing interests}

370 The authors declare that they have no competing interests.

\section{Author details}

$372{ }^{1}$ Department of Neurology, Affiliated ZhongDa Hospital, School of Medicine,

373 Southeast University, Nanjing, Jiangsu 210009, China. ${ }^{2}$ Shenzhen Institutes of 
Advanced Technology, Chinese Academy of Sciences. ${ }^{3}$ Department of Neurology, Nanjing Lishui people's Hospital, Nanjing, China. ${ }^{4}$ Department of Computer Science, University of Colorado Boulder.

\section{References}

1. Collaborators GBDN. Global, regional, and national burden of neurological disorders, 1990-2016: a systematic analysis for the Global Burden of Disease Study 2016. Lancet Neurol. 2019;18(5):459-80.

2. International AsD. World Alzheimer Report 20182018.

3. O'Brien JT, Thomas A. Vascular dementia. Lancet. 2015;386(10004):1698-706.

4. Larsson SC, Markus HS. Does Treating Vascular Risk Factors Prevent Dementia and Alzheimer's Disease? A Systematic Review and Meta-Analysis. J Alzheimers Dis. 2018;64(2):657-68.

5. Philip Scheltens KB, Monique M B Breteler, Bart de Strooper, Giovanni B Frisoni, Stephen Salloway, Wiesje Maria Van der Flier. Alzheimer's disease. Lancet 2016(388):505-17.

6. Rabin JS, Klein H, Kirn DR, Schultz AP, Yang HS, Hampton O, et al. Associations of Physical Activity and beta-Amyloid With Longitudinal Cognition and Neurodegeneration in Clinically Normal Older Adults. JAMA Neurol. 2019.

7. Wang R, Fratiglioni L, Kalpouzos G, Lovden M, Laukka EJ, Bronge L, et al. Mixed brain lesions mediate the association between cardiovascular risk burden and cognitive decline in old age: A population-based study. Alzheimers Dement. 2017;13(3):247-56.

8. Stewart RAH, Held C, Krug-Gourley S, Waterworth D, Stebbins A, Chiswell K, et al. Cardiovascular and Lifestyle Risk Factors and Cognitive Function in Patients With Stable Coronary Heart Disease. J Am Heart Assoc. 2019;8(7):e010641. 
9. van der Velpen IF, Feleus S, Bertens AS, Sabayan B. Hemodynamic and serum cardiac markers

397 and risk of cognitive impairment and dementia. Alzheimers Dement. 2017;13(4):441-53.

10. Heffernan KS, Augustine JA, Lefferts WK, Spartano NL, Hughes WE, Jorgensen RS, et al. Arterial stiffness and cerebral hemodynamic pulsatility during cognitive engagement in younger and older adults. Exp Gerontol. 2018;101:54-62.

11. Buratti L, Viticchi G, Falsetti L, Balucani C, Altamura C, Petrelli C, et al. Thresholds of impaired cerebral hemodynamics that predict short-term cognitive decline in asymptomatic carotid stenosis.

Journal of cerebral blood flow and metabolism : official journal of the International Society of Cerebral Blood Flow and Metabolism. 2016;36(10):1804-12.

12. Nielsen RB, Egefjord L, Angleys H, Mouridsen K, Gejl M, Moller A, et al. Capillary dysfunction is associated with symptom severity and neurodegeneration in Alzheimer's disease. Alzheimers Dement. 2017;13(10):1143-53.

13. Kim CM, Alvarado RL, Stephens K, Wey HY, Wang DJJ, Leritz EC, et al. Associations between cerebral blood flow and structural and functional brain imaging measures in individuals with neuropsychologically defined mild cognitive impairment. Neurobiol Aging. 2019.

14. Yew B, Nation DA, Alzheimer's Disease Neuroimaging I. Cerebrovascular resistance: effects on cognitive decline, cortical atrophy, and progression to dementia. Brain. 2017;140(7):1987-2001.

15. Firbank MJ, He J, Blamire AM, Singh B, Danson P, Kalaria RN, et al. Cerebral blood flow by arterial spin labeling in poststroke dementia. Neurology. 2011;76(17):1478-84.

16. Clark LR, Nation DA, Wierenga CE, Bangen KJ, Dev SI, Shin DD, et al. Elevated cerebrovascular resistance index is associated with cognitive dysfunction in the very-old. Alzheimers Res Ther. 2015;7(1):3. 

autoregulation in subjects with Alzheimer's disease, mild cognitive impairment, and controls: evidence

420 for increased peripheral vascular resistance with possible predictive value. J Alzheimers Dis. 2012;30(4):805-13.

18. Sabayan B, Jansen S, Oleksik AM, van Osch MJ, van Buchem MA, van Vliet P, et al.

Cerebrovascular hemodynamics in Alzheimer's disease and vascular dementia: a meta-analysis of transcranial Doppler studies. Ageing Res Rev. 2012;11(2):271-7.

19. Berman SE, Clark LR, Rivera-Rivera LA, Norton D, Racine AM, Rowley HA, et al. Intracranial Arterial 4D Flow in Individuals with Mild Cognitive Impairment is Associated with Cognitive Performance and Amyloid Positivity. J Alzheimers Dis. 2017;60(1):243-52. of fractional flow reserve from anatomic CT angiography. JAMA. 2012;308(12):1237-45. of Transluminal Attenuation Gradient and Noninvasive Fractional Flow Reserve Derived from 320Detector Row CT Angiography to Diagnose Hemodynamically Significant Coronary Stenosis: An NXT Substudy. Radiology. 2016;279(1):75-83.

22. Taylor CA, Fonte TA, Min JK. Computational fluid dynamics applied to cardiac computed tomography for noninvasive quantification of fractional flow reserve: scientific basis. J Am Coll Cardiol. 2013;61(22):2233-41. to Diagnose and Treat Cardiovascular Disease. Trends in biotechnology. 2017;35(11):1049-61. 
fluid dynamics tell us about intracranial aneurysms? A meta-analysis and critical review. Journal of cerebral blood flow and metabolism : official journal of the International Society of Cerebral Blood Flow and Metabolism. 2019:271678x19854640.

25. Nam HS, Scalzo F, Leng X, Ip HL, Lee HS, Fan F, et al. Hemodynamic Impact of Systolic Blood Pressure and Hematocrit Calculated by Computational Fluid Dynamics in Patients with Intracranial Atherosclerosis. J Neuroimaging. 2016;26(3):331-8.

26. Arzani A. Accounting for residence-time in blood rheology models: do we really need nonNewtonian blood flow modelling in large arteries? J R Soc Interface. 2018;15(146). waveforms derived from maximum Doppler spectral velocity. Ultrasound Med Biol. 2013;39(5):87081.

28. Scheel P, Puls I, Becker G, Schoning M. Volume reduction in cerebral blood flow in patients with vascular dementia. Lancet. 1999;354(9196):2137. correlates of transient cognitive impairment after transient ischemic attack and minor stroke: A transcranial Doppler study. Int J Stroke. 2016;11(9):978-86. intracranial venous blood flow and pulsatility in Alzheimer's disease: A 4D flow MRI study. Journal of

458 cerebral blood flow and metabolism : official journal of the International Society of Cerebral Blood 459 Flow and Metabolism. 2017;37(6):2149-58. 
462 Alzheimer disease and mild cognitive impairment: a marker for disease severity. Radiology.

$463 \quad 2013 ; 267(1): 221-30$.

464 32. Boban M, Crnac P, Junakovic A, Garami Z, Malojcic B. Blood flow velocity changes in anterior

465 cerebral arteries during cognitive tasks performance. Brain Cogn. 2014;84(1):26-33.

466 33. Schuff N, Matsumoto S, Kmiecik J, Studholme C, Du A, Ezekiel F, et al. Cerebral blood flow in

467 ischemic vascular dementia and Alzheimer's disease, measured by arterial spin-labeling magnetic

468 resonance imaging. Alzheimers Dement. 2009;5(6):454-62.

469 34. Nation DA, Wierenga CE, Clark LR, Dev SI, Stricker NH, Jak AJ, et al. Cortical and subcortical

470 cerebrovascular resistance index in mild cognitive impairment and Alzheimer's disease. J Alzheimers

$471 \quad$ Dis. 2013;36(4):689-98.

472 35. Nortley R, Korte N, Izquierdo P, Hirunpattarasilp C, Mishra A, Jaunmuktane Z, et al. Amyloid

473 beta oligomers constrict human capillaries in Alzheimer's disease via signaling to pericytes. Science.

$474 \quad 2019 ; 365(6450)$.

475 


\section{Figures}

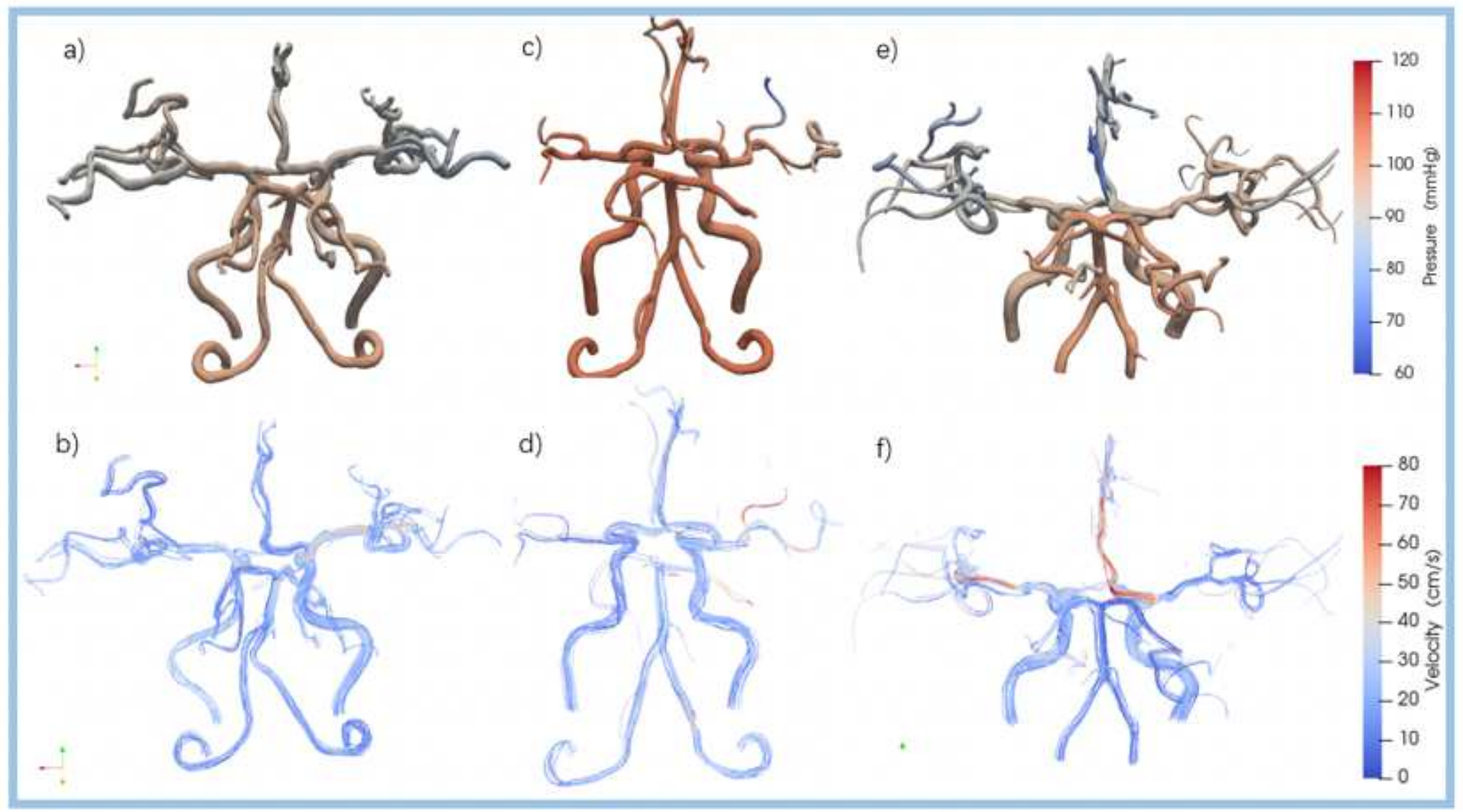

\section{Figure 1}

Three typical examples of pressure distribution and stream lines of blood flow velocity are displayed in the first and the second row, respectively. The first column( fig a and $b$ ) is for an AD patients, the second column (fig $c$ and d ) is for a VaD patients, and the third column (fig e and f) is for a healthy subject. It is evident that the AD patient and the healthy subject are with intact arterial trees, whereas VaD the patient is with scarce arterial branches. However, according to computation, the total blood flow in the models was $692 \mathrm{ml} / \mathrm{min}$ (AD patient), $647 \mathrm{ml} / \mathrm{min}$ (VaD patient), and $998 \mathrm{ml} / \mathrm{min}$ (healthy subject) respectively. 
a)

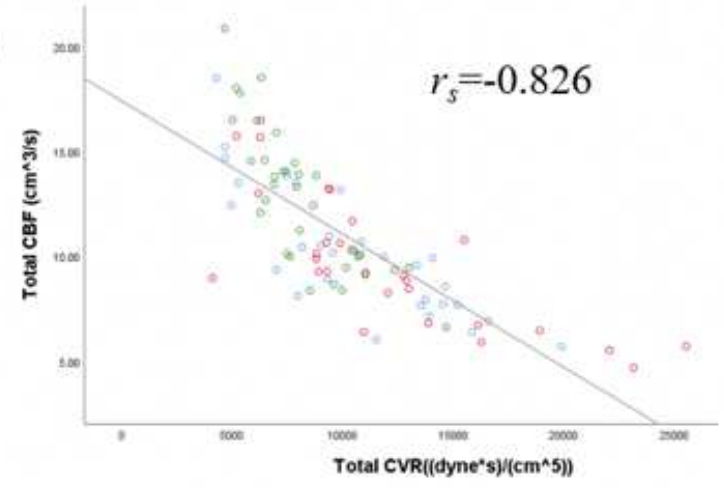

c)
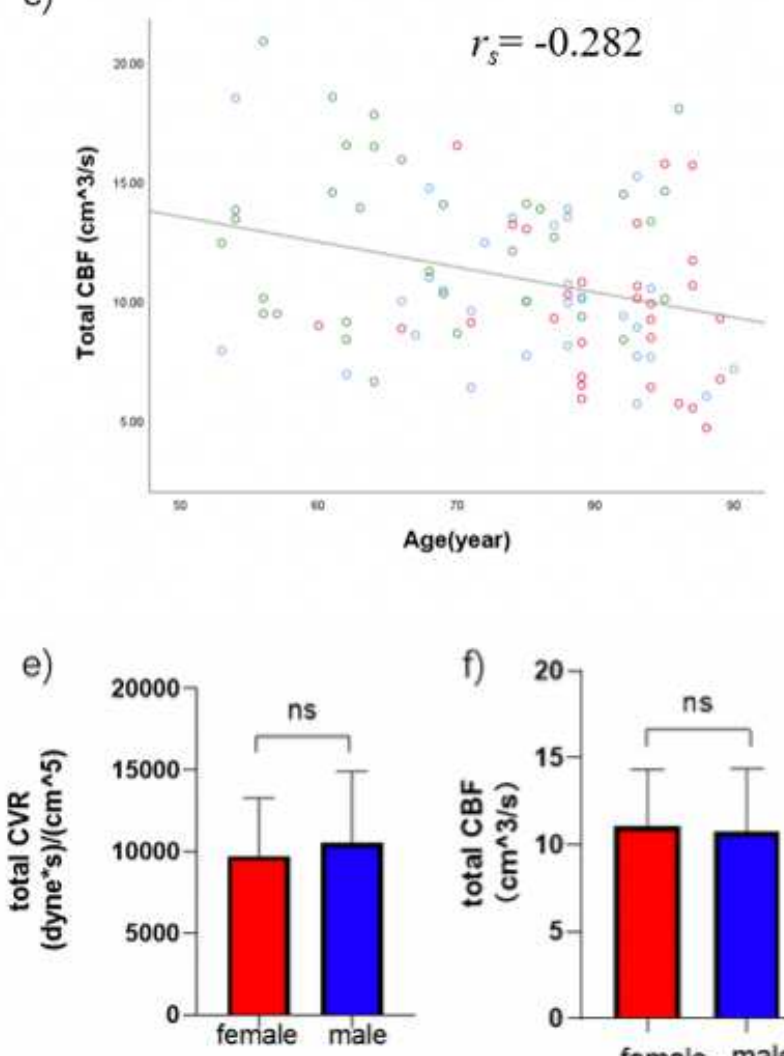

b)

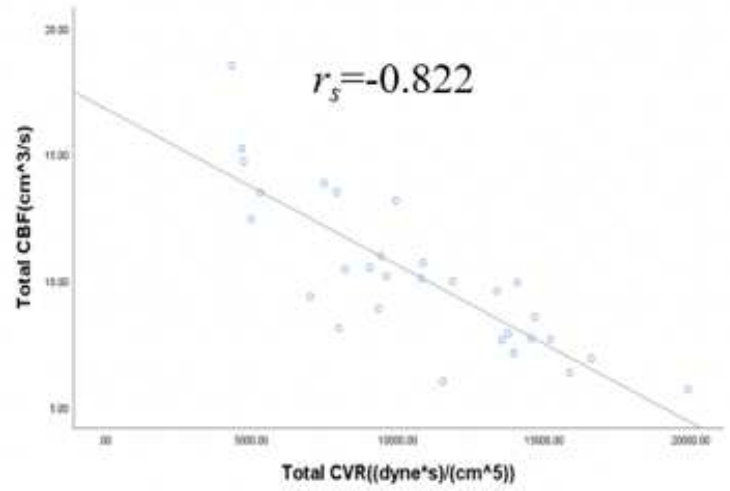

d)

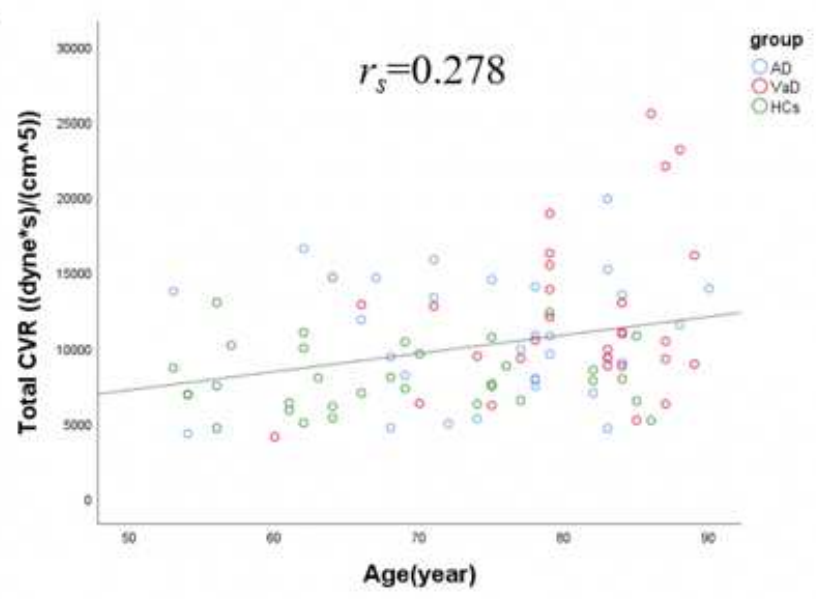

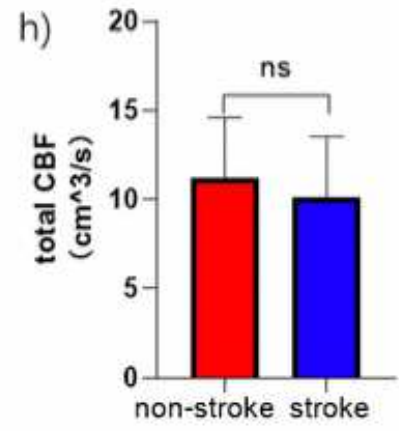

Figure 2

Interactive associations of the hemodynamic parameters and risk factors, correlation between total CBF and CVR in all subjects (a) and AD group(b), (c) and (d) showed significant correlations between CBF or CVR and age, fig $(e, f)$ showed there were no significant difference of total CVR or CBF between male and female patients, fig $(\mathrm{g})$ indicated there was significant difference of total CVR in patients with stroke or not, but not total CBF fig(h).ns: no significance, * $\mathrm{P}<0.05$.

\section{Supplementary Files}

This is a list of supplementary files associated with this preprint. Click to download. 
- supplementarymaterial.docx 